Interfaces and Free Boundaries 2, (2000) 1-19

\title{
Existence and uniqueness of a classical solution for a mathematical model describing the isobaric crystallization of a polymer
}

\author{
A. FASANO ${ }^{\dagger}$ \\ Università degli Studi di Firenze, Dip. Matematica “U. Dini”, viale Morgagni n. 67A, 50134 \\ Firenze \\ AND \\ A. MANCINI ${ }^{\ddagger}$ \\ Università degli Studi di Milano, Dip. Matematica "F. Enriques", via Saldini n. 50, 20122 Milano \\ [Received 19 September 1998 and in revised form 19 July 1999]

\begin{abstract}
In this paper a global existence and uniqueness result is presented for the classical solution of a free boundary problem for a system of partial differential equations (p.d.e.s) with non-local boundary conditions describing the crystallization process of a cylindrical sample of polymer under prescribed pressure. The system of equations is discussed in [16] as the model for coupled cooling and shrinking of a sample of molten polymer under a given constant pressure. The velocity field generated by the thermal and chemical contraction enters the model only through its divergence. Such an approximation is discussed on the basis of a qualitative analysis.
\end{abstract}

Keywords: Free boundary problems; nonlinear parabolic equations; phase change.

\section{Introduction}

It is well known (see e.g. [1], [3], [5], [10], [13]-[15], [26], [30]) that phase change in polymers is a process of high complexity due to several reasons: (i) polymers crystallize not as pure crystals but in structures having a roughly spherical lamellar organization with amorphous inclusions (spherulites); (ii) spherulites can nucleate and grow over a temperature interval $\left(T_{g}, T_{m}\right)$ depending on pressure, with a rate also depending on temperature and pressure, as well as on the volume fraction of the crystals already formed; (iii) a maximum attainable crystal volume fraction $\left(w_{e q}\right)$ is defined at any given temperature $T \in\left(T_{g}, T_{m}\right)$.

For the literature on theoretical aspects of crystallization see in particular the survey paper [5], while [10] contains several details on the experimental quantities.

In addition there is a well-developed theory based on probabilistic arguments (see [9], [28] and the references therein).

The aim of the present paper is the study of a free boundary problem with nonlocal boundary conditions modeling the solidification of a sample of molten polymer under a given constant pressure. The model predicts the evolution of the temperature $T$, the crystal volume fraction $w$ and the height of the sample $h$, which varies owing to thermal contraction and crystal growth.

\footnotetext{
†Email: fasano@udini.math.unifi.it

\$Email: mancini@ares.mat.unimi.it
} 
The experimental work has been performed by Montell and the model has been formulated in cooperation with Dr S .Mazzullo of Montell. For a general discussion about the modeling aspects we refer to the forthcoming paper [16], although in Section 2 we will provide enough information to understand the physics of the process.

In this paper we are more interested in the problem of the well posedness of the model, while we refer to [23] for the numerical calculations performed on a more complete version, which show an excellent agreement with the experiments and at the same time suggest the simplifications adopted here.

It has to be remarked that the model has proved to be not just a predicting tool, but it helped to point out many relevant physical features of the phenomenon, such as: (a) in what temperature region the choice of the crystallization kinetics is important, (b) in what interval the process is dominated by the so-called equilibrium crystallization $w_{e q}(T)$ (see Fig. 1), (c) what type of temperature dependence can be assumed for the latter quantity (see Fig. 2), (d) how large the influence of the thermocouple introduced in the system is.

We will prove existence and uniqueness of a classical solution globally in time.

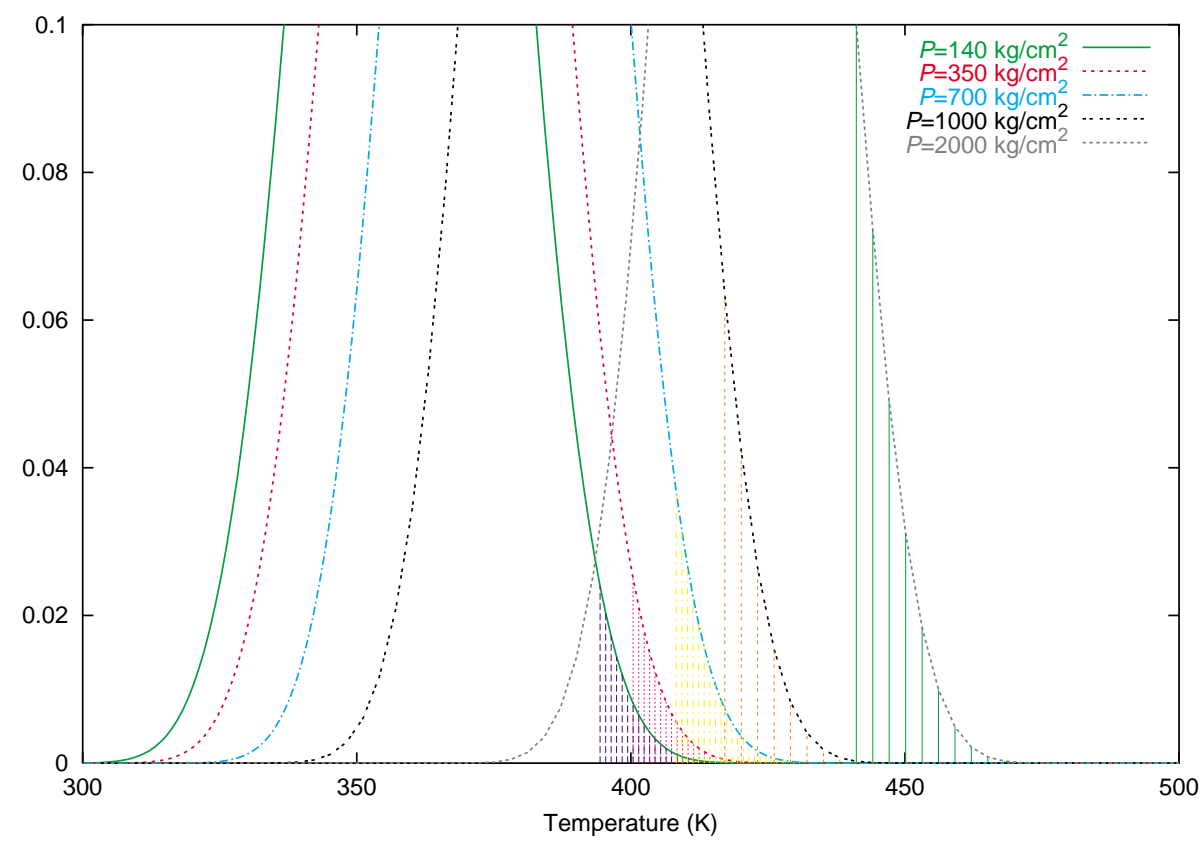

FIG. 1. Fraction of the crystallization temperatures range where the effect of the kinetics is operative (dashed zone).

\section{Formulation of the model}

The domain occupied by the molten polymer at $t=0$ is a cylinder (typical size: height $=7$ $\mathrm{cm}$, radius $=0.5 \mathrm{~cm}$ ) provided with a metallic piston which produces the desired pressure. On 
the cylinder axis a thermocouple is placed supported by a thin metallic rod whose length is approximately one-third of the cylinder length. The bottom of the cylinder is insulated. Heat loss through the lateral walls follows a law of linear radiation, while the metallic bodies of the piston and of the thermocouple rod are regarded as concentrated capacities, since their thermal conductivity is much larger than that of the polymer. Initially the polymer temperature $T_{0}$ is uniform and above the melting point.

\subsection{The governing differential equations}

We have to find the temperature $T$, the crystal volume fraction $w$, and the motion of the piston.

The system of p.d.e.'s we are going to consider is the following:

$$
\begin{aligned}
\rho C T_{t} & =k \Delta T+\rho_{c} L w_{t}, \\
w_{t}+w \operatorname{div} \vec{V} & =W(P, T, w), \quad w<w_{e q}(T) \\
\operatorname{div} \vec{V} & =-\frac{1}{\rho(T, w)}\left[\frac{\partial \rho}{\partial T} T_{t}+\frac{\partial \rho}{\partial w} w_{t}\right]=-\frac{\partial}{\partial t}\left(\ln \frac{\rho}{\rho_{0}}\right)
\end{aligned}
$$

where $C$ is the specific heat, $k$ the thermal conductivity, and $L$ the latent heat of crystallization. The density $\rho$ depends on $T, w$ and on pressure and is a $C^{2}$ function with Lipschitz continuous second derivatives, while $\rho_{c}$ is defined as the value of $\rho$ for $w=1$ (i.e. the density of pure crystals). Finally, $\rho_{0}$ is a reference density (e.g. $\rho_{0}=\rho\left(P, T_{m}, 0\right)$ ).

We will comment soon the particular form of the equations above. Equation (2.1) expresses heat balance and includes the heat released in the sample during crystallization. Equation (2.2) describes the evolution of the crystal volume fraction, confined to the interval in which $w$ is below the threshold $w_{e q}$, whose dependence on $P$ is not emphasized. Here the function $W$ represents the crystallization rate and will be specified below. The last equation expresses the fact that the specific volume of the system varies because $T$ and $w$ depend on time and only for that reason. The latter statement is in agreement with the fact that the system is practically incompressible in the range of pressure experienced during the process. As a matter of fact (2.1)-(2.3) is a simplification of a more complete model including the convective terms $\rho C \vec{V} \cdot \nabla T$ in (2.1), $\vec{V} \cdot \nabla w$ in (2.2) and the flow equations for the system, considered as a (highly) viscous fluid whose viscosity depends on $T$ and $w$. Numerical simulations [23] have shown that with excellent approximations:

(i) pressure can be taken constant throughout the sample (this justifies (2.3)),

(ii) the effect of convection in the equations for $T$ and $w$ is negligible, so that the operator $\frac{\partial}{\partial t}$ replaces the Lagrangian derivative everywhere;

(iii) the only term connected with the thermally induced flow having some relevance during the process is $w \operatorname{div} \vec{V}$, appearing in (2.2).

The source term $W(P, T, w)$ in (2.2) expresses the crystallization kinetics and is chosen as

$$
W(P, T, w)=B(P, T) w^{a}\left(w_{e q}-w\right)^{b}, \quad w<w_{e q}(T),
$$




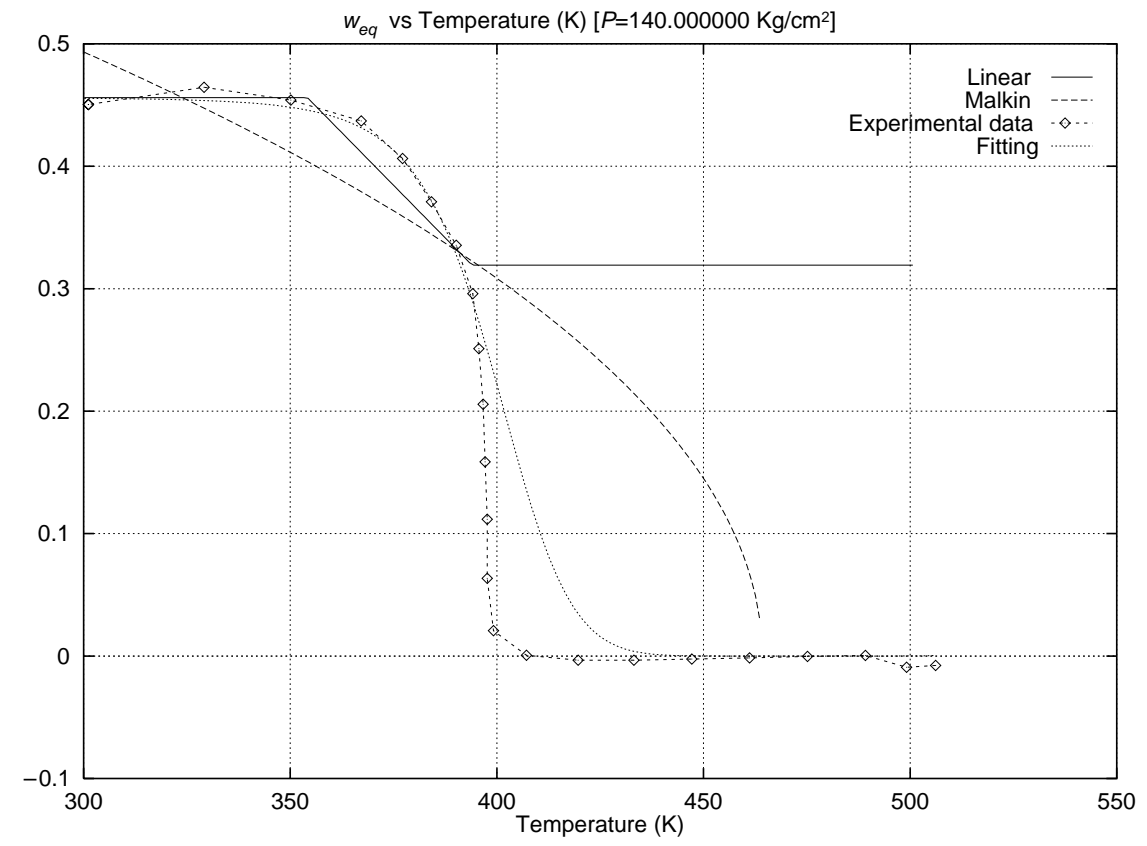

FIG. 2. Different models for $w_{e q}$ compared with the measured mean crystallinity.

where $B$ is a continuous bell-shaped function supported in the temperature interval $\left(T_{g}(P), T_{m}(P)\right)$. Typical values for the exponents $a, b$ are $a=2 / 3, b=0.765$ (all numerical values refer to the specific case of polypropylene $)^{\dagger}$.

The function $w_{e q}(P, T)$ is defined for each $P$ in the support if $B$ is a Lipschitz continuous decreasing function of $T$. The presence of the constraint $w_{e q}$ in (2.4) is a basic feature of polymer crystallization and it turns out to be extremely crucial for polypropylene.

We have to incorporate in the model a smooth transition between the regime governed by (2.4) and the action of the obstacle $w=w_{e q}$. A simple way to do this will be illustrated in Section 6 and consists of modifying (2.4) for $w$ in a small interval near $w_{e q}$ so that $w_{t}$ is not discontinuous when $w$ hits $w_{e q}$.

Although such a procedure is suggested by mathematical arguments, it has, nevertheless, a quite acceptable physical explanation. Indeed the sharp cut on $w$ represented by $w_{e q}$ is a crude representation of reality. We can understand this claim by looking at the complex structure of spherulites, whose amorphous component is responsible for the presence of $w_{e q}$. We can say that crystallization proceeds first through a regime of massive growth of the main (radial) lamellae and that it reaches the maximum crystallinity through a slower process of secondary crystallization (branching).

$\dagger$ The kinetic law (2.4) resembles the one proposed in [8], but with different values for the exponents $a$ and $b$.

A discussion about crystallization kinetic laws of the form $w_{t}=F(T) G(w)$ can be found in [5], [18], and [19]. It is shown in [5] and [19] that they are the only laws consistent with the so-called additivity rule and in [18] that they admit traveling wave solutions. 
Thus we shall proceed as follows.

First we complete the formulation of the problem with the initial and boundary conditions, then we proceed to proving existence and uniqueness for a sufficiently small time interval in which $w$ is far enough from $w_{e q}$. Then we show that the solution can be extended to infinity introducing a smooth transition between the two regimes of crystallization.

REMARK 2.1 Since we are considering isobaric cooling processes, from now on we will drop the symbol $P$ in all formulas.

REMARK 2.2 An important feature to keep in mind is that, consistent with the fact that convection has no role in the evolution of $T$ and $w$, we can adjust the formulation of the model by inserting convective terms which render its structure mathematically more convenient. The existence proof is in fact concerned with the problem reformulated in Section 4 in a fixed domain, which is physically equivalent to the one we are describing here following the simple criterion of neglecting convection altogether.

Equations (2.1)-(2.3) must be solved in the moving domain

$$
R_{\theta}=\left\{(r, z, t) \in \mathbb{R}^{3} \mid(r, z, t) \in \Omega_{t}, 0<t<\theta\right\}
$$

with $\Omega_{t}$, the cylinder defined by the conditions

$$
(r, z) \in \Omega_{t} \text { iff }\left\{\begin{array}{l}
r_{c}<r<R \\
0<r<R
\end{array} \quad \text { and } \quad \begin{array}{c}
0<z<l, \\
0<z<h(t),
\end{array}\right.
$$

being $R, l, r_{c}$ known quantities ( $R$, radius of the cylindrical sample; $l, r_{c}$, height and radius of the thermocouple support) and $h(t)$ unknown.

\subsection{Boundary conditions for the thermal field}

As we said, the initial conditions are

$$
T(r, z, 0)=T_{0}>T_{m} \quad \text { everywere in } \Omega_{0} .
$$

At the bottom surface $\Gamma_{b}$ (where the presence of the thermocouple can be disregarded) we take

$$
T_{z}(r, 0, t)=0, \quad \text { on } \Gamma_{b}, \quad 0<t<\theta .
$$

On the lateral surface $\Gamma_{e}$ we have

$$
-k T_{r}(R, z, t)=\phi_{e}\left(T-T_{e}\right), \quad 0<z<h(t), \quad 0<t<\theta,
$$

where $T_{e}<T_{g}$ is the outside temperature and $\phi_{e}$ is a positive constant.

On the piston surface $\Gamma_{p}$ we write

$$
-k T_{z}(r, h(t), t)=\phi_{p}\left(T-T_{p}(t)\right), \quad 0<r<R, \quad 0<t<\theta,
$$


where $T_{p}(t)$ is the still unknown temperature of the piston and $\phi_{p}>0$ and constant.

A similar condition holds on the boundary $\Gamma_{c}$ of the thermocouple support (radius $r_{c} \ll R$, length $l \simeq h_{0} / 3$ ).

Since the heat exchange through the tip of the thermocouple is negligibly small in comparison with the whole heat flow trough the support, we may smooth the support boundary $\Gamma_{0 c}$ to $\Gamma_{0 c}^{\varepsilon}$ in some arbitrary way. So we write the following boundary condition

$$
-k \nabla T \cdot \vec{n}=\phi_{0 c}^{\varepsilon}(z)\left(T-T_{c}(t)\right) \text { on } \Gamma_{0 c}^{\varepsilon},
$$

$\vec{n}$ being the outside normal to the boundary $\Gamma_{0 c}^{\varepsilon}$,

$$
\Gamma_{0 c}^{\varepsilon}= \begin{cases}\left(r_{c}, z\right), & 0<z<l-\varepsilon \\ (\mu(z), z), & l-\varepsilon \leqslant z \leqslant l+\varepsilon \\ (0, z), & l+\varepsilon<z<h(t)\end{cases}
$$

and

$$
\phi_{0 c}^{\varepsilon}(z)= \begin{cases}\phi_{c}, & 0<z<l-\varepsilon \\ \phi_{0}, & l-\varepsilon \leqslant z \leqslant l+\varepsilon \\ 0, & l+\varepsilon<z<h(t)\end{cases}
$$

for smooth monotone functions $\mu(z)\left(\mu(l-\varepsilon)=r_{c}, \mu(l+\varepsilon)=0\right)$ and $\phi_{0}(z)\left(\phi_{0}(l-\varepsilon)=\phi_{c}\right.$, $\left.\phi_{0}(l+\varepsilon)=0\right)$.

The evolution of temperatures $T_{p}, T_{c}$ is determined by the balance equations

$$
\begin{gathered}
C_{p} \dot{T}_{p}(t)=-k \int_{0}^{R} 2 \pi r T_{z}(r, h(t), t) \mathrm{d} r, \quad 0<t<\theta, \\
C_{c} \dot{T}_{c}(t)=k 2 \pi r_{c} \int_{0}^{l} T_{r}\left(r_{c}, z, t\right) \mathrm{d} z, \quad 0<t<\theta,
\end{gathered}
$$

with the initial conditions $T_{p}(0)=T_{c}(0)=T_{0}, C_{p}, C_{c}$ being the respective heat capacities.

Note that in (2.11) we have neglected the heat exchange through the tip of the thermocouple.

Inserting (2.8) in (2.10) we obtain a linear ordinary differential equation (o.d.e.) for $T_{p}$ which gives:

$$
T_{p}(t ; T, h)=e^{-\lambda_{p} t}\left[T_{0}+\frac{2 \lambda_{p}}{R^{2}} \int_{0}^{t}\left(e^{\lambda_{p} s} \int_{0}^{R} r T(r, h(s), s) \mathrm{d} r\right) \mathrm{d} s\right]
$$

with $\lambda_{p}=\frac{\pi \phi_{p} R^{2}}{C_{p}}$.

Likewise we obtain

$$
T_{c}(t ; T)=e^{-\lambda_{c} t}\left[T_{0}+\frac{\lambda_{c}}{l} \int_{0}^{t}\left(e^{\lambda_{c} s} \int_{0}^{l} T\left(r_{c}, z, s\right) \mathrm{d} z\right) \mathrm{d} s\right]
$$

with $\lambda_{c}=\frac{2 \pi r_{c} \phi_{c} l}{C_{c}}$.

Equations (2.12), (2.13) must be put back into (2.8), (2.9). 
REMARK 2.3 In the thermal problem we have taken $k$ and $C$ to be constant for the sake of simplicity, but it would not be too difficult to let them depend on $T, w$. Another possible generalization is to replace (2.7)-(2.9) with non-linear radiation laws.

Introducing the functions $\Phi$ and $T_{e x t}$ defined on the boundary of $D_{\theta}$ as:

$$
\begin{gathered}
\Phi(r, z, t)= \begin{cases}\phi_{0 c}^{\varepsilon} & \text { on } \Gamma_{0 c}^{\varepsilon} \\
0 & \text { on } \Gamma_{b} \\
\phi_{e} & \text { on } \Gamma_{e} \\
\phi_{p} & \text { on } \Gamma_{p}\end{cases} \\
T_{e x t}\left(r, z, t ; T_{c}, T_{p}\right)= \begin{cases}T_{c} & \text { on } \Gamma_{c} \cup \Gamma_{0} \\
0 & \text { on } \Gamma_{b} \\
T_{e} & \text { on } \Gamma_{e} \\
T_{p} & \text { on } \Gamma_{p}\end{cases}
\end{gathered}
$$

we can write boundary condition for the thermal field in the following compact form

$$
-k \nabla T \cdot \vec{n}=\Phi\left(T-T_{\text {ext }}\right) \quad \text { on } \Gamma .
$$

\subsection{Initial condition for $w$}

The elimination of the term $\nabla w \cdot \vec{V}$ in the equation for $w$ reduces it to an o.d.e., although quite non-trivial due to the presence of $\operatorname{div} \vec{V}$. We shall see how to deal with (2.2). The only data we need to know are for $t=0$ :

$$
w(r, z, 0)=0, \quad \text { in } D_{0} .
$$

2.4 The free boundary $z=h(t)$

The height of the cylinder is defined by mass conservation:

$$
2 \pi \int_{0}^{h(t)} \int_{0}^{R} r \rho(T, w) \mathrm{d} r \mathrm{~d} z=m
$$

$m$ being the total mass of the sample, condition (2.18) is obviously nonlocal.

\section{Statement of the problem}

Problem (P). Find a triple $(T, w, h)$ satisfying (2.1), (2.5), (2.9), (2.12), (2.18) in some time interval $(0, \theta)$ in the classical sense. By solution of $(2.2)$ we mean a maximal solution.

A condition on $\rho(T, w)$ which plays an important role is the following

$$
\frac{\rho_{w}}{\rho} w \leqslant K<1, \quad \forall w \in[0,1], \quad T \in\left[T_{e}, T_{0}\right] .
$$


We will show that problem $P$ has one unique solution in a suitable time interval $(0, \theta)$ in the Hölder space $H^{2+a, 1+a / 2}$ (see Theorem 5.1). Next we will extend such a solution to all times in Section 6.

REMARK 3.1 Actually the existence theorem refers to the modified model illustrated in section 3, which differs from the one just stated only by some convective terms whose contribution, consistent with the approximations adopted so far, is immaterial (see Remark 4.4).

REMARK 3.2 A specific form for $\rho(P, T, w)$ is:

$$
\frac{1}{\rho}=\omega+\frac{(1-w)}{p+\pi} R^{*}\left(T-T_{0}\right)
$$

where $\omega, \pi, R^{*}, T_{0}$ are physical parameters of the material and it can be checked that, for the data of polypropylene, condition (3.1) is satisfied.

\section{Reformulation of the problem and some a priori results}

First of all we eliminate $w_{t}$ from (2.1). This step is necessary in order to find the condition guaranteeing the parabolicity of (2.1), since $w_{t}$ is linked to $T_{t}$ through (2.2), (2.3).

In what follows we just suppose $w<w_{e q}$. Let us rewrite (2.1)-(2.3) in the form

$$
\left(\begin{array}{ccc}
\rho C & -\rho_{c} L & 0 \\
0 & 1 & w \\
\rho_{T} & \rho_{w} & \rho
\end{array}\right)\left(\begin{array}{c}
T_{t} \\
w_{t} \\
\operatorname{div} \vec{V}
\end{array}\right)=\left(\begin{array}{c}
k \Delta T \\
W(T, w) \\
0
\end{array}\right),
$$

from which we deduce

$$
T_{t}=A_{0}(T, w) \Delta T+A_{1}(T, w) W(T, w)
$$

with

$$
\begin{aligned}
A_{0}(T, w) & =\frac{\left(1-w \hat{\rho}_{w}\right)}{\mathcal{Z}} k, \quad A_{1}(T, w)=\frac{\rho_{c} L}{\mathcal{Z}}, \\
\mathcal{Z} & =\rho C\left[1-w \hat{\rho}_{w}-\frac{\hat{\rho}_{c} \hat{\rho}_{T} L}{C} w\right], \\
\hat{\rho}_{w} & =\frac{\rho_{w}}{\rho}, \quad \hat{\rho}_{T}=\frac{\rho_{T}}{\rho} .
\end{aligned}
$$

Therefore parabolicity is guaranteed by (3.1):

PROPOSITION 4.1 Equation (4.1) is uniformly parabolic if (3.1) holds.

From now on we replace (2.1) with (4.1).

Then we transform (2.2), eliminating $\operatorname{div} \vec{V}$ and writing it in the form

$$
\frac{\partial}{\partial t} \ln \frac{w}{\rho / \rho_{0}}=B w^{a-1}\left(w_{e q}-w\right)^{b}
$$


from which we deduce the following integral equation for $w$

$$
w=\rho(T, w)\left\{(1-a) \int_{0}^{t} \rho^{a-1}(T, w) B(T)\left(w_{e q}-w\right)^{b} \mathrm{~d} t\right\}^{\frac{1}{1-a}}
$$

were we assumed for the sake of simplicity $\rho_{0}=1$.

An a priori result can be obtained as a consequence of the maximum principle for the thermal problem:

Proposition 4.2 During the whole process we have $T_{e} \leqslant T \leqslant T_{0}$.

Proof. If $T$ takes a maximum larger than $T_{0}$ at some time $\hat{t}$, then the source term in (4.1) will vanish in a neighborhood of such a maximum point, which for this reason must be located on the boundary. We can exclude immediately the subset $\Gamma_{b} \cup \Gamma_{e}$. Suppose $T=T_{\max }>T_{0}$ on $\Gamma_{p}$. Then because of (2.8) and Hopf's principle we must have $T_{p}(\hat{t})>T_{\max }$ and from $(2.8),(2.10), \dot{T}_{p}(\hat{t})<0$. Therefore there has to be some $\tilde{t}<\hat{t}$ in which $T_{p}(\tilde{t})>T_{p}(\hat{t})$ and $\dot{T}_{p}(\tilde{t}) \geqslant 0$. The latter inequality contradicts (2.10), since for $t=\tilde{t}$ (2.8) implies $-k T_{z}<0$ everywhere on $z=h$. In a similar way we exclude that the maximum can be taken on $\Gamma_{c}$. A parallel argument shows that $T \geqslant T_{e}$.

We conclude this section by introducing some minor change in the model. First of all we remark that it is convenient to deal with a domain which does not depend on time. For this reason, given a smooth function $\psi(\zeta, h(t)) \geqslant 0$ such that $\psi \equiv 0$ for $\zeta \leqslant 0$ and $\psi\left(h(t)-\frac{h_{0}}{2}, h(t)\right)=1$, we introduce the change of variable

$$
y=z+\left(h_{0}-h(t)\right) \psi\left(z-\frac{h_{0}}{2}, h(t)\right)
$$

which leaves the domain unchanged for $z \leqslant \frac{h_{0}}{2}$ and maps the remaining part onto the cylinder $0<r<R, \frac{h_{0}}{2}<y<h_{0}, 0<t<\theta$. In this way the new boundary is fixed in time. We will denote the new domain by $D_{\theta}$.

Therefore defining the unknowns

$$
\tilde{T}(r, y, t)=T(r, z, t), \quad \tilde{w}(r, y, t)=w(r, z, t),
$$

(4.1) becomes $\left(\tilde{T}_{t} \simeq T_{t}\right.$, see Remark 4.3)

$$
\tilde{T}_{t}=A_{0}(\tilde{T}, \tilde{w})\left[\Delta_{h} \tilde{T}+\tilde{T}_{y}\left(h_{0}-h\right) \psi_{\zeta \zeta}\right]+A_{1}(\tilde{T}, \tilde{w}) W(\tilde{T}, \tilde{w})
$$

where $\Delta_{h}$ is the Laplacian operator in $(r, y)$ variables with the addition of the term $\left[\frac{\partial y}{\partial z}\right]^{2}$ in front to $T_{y y}$.

REMARK 4.3 When applying transformation (4.7) we neglect the terms in $\dot{h}$ appearing in the differential equation, consistent with the approximation of suppressing convection. 
In the same spirit we keep the form (2.2), i.e. (4.5) for the equation to be satisfied by $\tilde{w}$ implying (by means of (4.6))

$$
\tilde{w}=\rho(\tilde{T}, \tilde{w})\left\{(1-a) \int_{0}^{t} \rho^{a-1}(\tilde{T}, \tilde{w}) B(\tilde{T})\left(w_{e q}-\tilde{w}\right)^{b} \mathrm{~d} t\right\}^{\frac{1}{1-a}} .
$$

The equation for the free boundary becomes

$$
2 \pi \int_{0}^{h_{0}} \int_{0}^{R} r \rho(\tilde{T}, \tilde{w})\left(1+\left(h_{0}-h\right) \psi_{\zeta}\right) \mathrm{d} r \mathrm{~d} y=m,
$$

and the boundary condition for $\tilde{T}$ has the form

$$
\begin{aligned}
& -k \nabla_{h} \tilde{T} \cdot \vec{n}=\tilde{\Phi}(r, y, t)\left(\tilde{T}-\tilde{T}_{e x t}\left(r, y, t ; \tilde{T}_{c}, \tilde{T}_{p}\right)\right) \text { on } \\
& \qquad=\partial D_{\theta} \backslash\{(r, z, t) \mid t=0, t=\theta\}
\end{aligned}
$$

with obvious definitions of the functions $\tilde{\Phi}, \tilde{T}_{\text {ext }}, \tilde{T}_{c}, \tilde{T}_{p}$.

REMARK 4.4 Equations (4.8) and (4.9) do in fact replace (2.1), i.e. (4.1), and (2.2), i.e. (4.6).

REMARK 4.5 A final change introduced in the model is that the domain $D_{\theta} \cap\{t=$ const. $\}$ is approximated in a standard way by a set with a smooth boundary, say $D_{\theta}^{\varepsilon}$. This implies that we have to define a new couple of functions $\Phi^{\varepsilon}, T_{e x t}^{\varepsilon}$ defined on the new smooth boundary and approximating smoothly $\Phi^{\varepsilon}$ and $T_{e x t}^{\varepsilon}$.

\section{Local existence and uniqueness theorem}

THEOREM 5.1 Under the regularity assumptions specified for $\rho(T, w)$ for a suitably small $\theta$ there exists a unique solution $(\tilde{T}, \tilde{w}, h)$ of $(4.8),(4.9),(4.10)$ with boundary condition (4.11) and the initial condition

$$
\tilde{T}(r, y, 0)=T_{0}
$$

Under these conditions the function $\tilde{T}$ belongs to the Hölder space $\mathcal{H}^{2+\alpha, 1+\frac{\alpha}{2}}$.

REMARK 5.2 We will deal with the continuation of the solution in section 6.

REMARK 5.3 In (4.11) the functions $T_{c}$ and $T_{p}$ are defined by means of (2.12), (2.13).

REMARK 5.4 The proof of the theorem is performed assuming a smooth domain with smooth boundary conditions in the sense of Remark 4.5. Here we keep the simpler notation $D_{\theta}$ (instead of $D_{\theta}^{\varepsilon}$ ) but we mean that $\partial D_{\theta}$ is smooth.

REMARK 5.5 The smoothing of the domain and boundary conditions is a minor modification on the physics of the model (if it is a modification at all) and it simplifies considerably the mathematical analysis. However, we remark that we could deal directly with the original domain using the estimates of weighted Hölder norms obtained for the solution of a parabolic equation in a wedge by 
V.A. Solonnikov in [29] $]^{\dagger}$. In order to be specific we describe how the smoothing can be performed, confining ourselves to the modifications near the border of the piston $(z=h(t), r=R)$. Let $H_{\varepsilon}(\xi)$ be any $C^{\infty}$ approximation of the Heaviside function such that $H_{\varepsilon}(\xi)=0$ for $\xi<0, H_{\varepsilon}(\xi)=1$ for $\xi>\varepsilon$ and $H_{\varepsilon}^{\prime}(\xi)>0$ for $\xi \in(0,1)$. Then the sharp edge at the piston boundary is modified by taking the axisymmetric surface $z=h-\varepsilon H_{\varepsilon}(r-R-\varepsilon)$. The function $T_{e x t}^{\varepsilon}$ and $\phi_{\varepsilon}$ can be defined along the modified boundary as

$$
T_{\text {ext }}^{\varepsilon}=\delta_{\varepsilon}(z) T_{e}+\left[1-\delta_{\varepsilon}(z)\right] T_{p}^{\varepsilon}, \quad \phi_{\varepsilon}=\delta_{\varepsilon}(z) \phi_{e}+\left[1-\delta_{\varepsilon}(z)\right] \phi_{p},
$$

with $\delta_{\varepsilon}(z)=H_{\varepsilon}(h-z)$, and $T_{p}^{\varepsilon}$ is obtained by replacing $R$ with $R-\varepsilon$ in (2.12). A similar procedure can be used to regularize the other parts of the boundary.

Proof of Theorem 5.1. The proof of Theorem 5.1 is based on a fixed point argument.

Let us first define the set

$$
\begin{gathered}
\mathcal{B}=\left\{(\tau, \omega, \chi) \mid\|\tau\|_{0} \leqslant M_{1},\|\tau\|_{\mathcal{H}^{2+\alpha, 1+\frac{\alpha}{2}}} \leqslant M_{2},\|\tau\|_{\mathcal{H}^{\alpha, \frac{\alpha}{2}}} \leqslant M_{3},\right. \\
\tau(r, y, 0)=T_{0}, \tau_{t}(r, y, 0)=0, \frac{1}{2} T_{e} \leqslant \tau \leqslant 2 T_{0} \\
\sup _{D_{\theta}}|\nabla \omega| \leqslant N_{1}, \sup _{D_{\theta}}\left|\omega_{t}\right| \leqslant N_{2}, \omega(r, y, 0)=0, \\
\omega(r, y, t) \leqslant w_{e q}(T)-\delta,\|\chi\|_{\mathcal{H}^{\frac{1}{2}+\frac{\alpha}{2}}}(0, \theta) \leqslant Q, \\
\left.\chi(0)=h_{0}=\frac{m}{\pi R^{2} \rho\left(T_{0}, 0\right)}, l<\frac{h_{0}}{2}<h_{\min }=\frac{m}{\pi R^{2} \rho_{\max }} \leqslant \chi \leqslant h_{0}\right\},
\end{gathered}
$$

where the constants $M_{i}$ have to be chosen and $\delta$ is given less than the inf of $w_{e q}(\tau)$ in the range of $\tau$.

Now we define the functions

$$
\begin{aligned}
& \tau_{p}(t)=e^{-\lambda_{p} t}\left[T_{0}+\frac{2 \lambda_{p}}{R^{2}} \int_{0}^{t}\left(e^{\lambda_{p} s} \int_{0}^{R} r \tau\left(r, h_{0}, s\right) \mathrm{d} r\right) \mathrm{d} s\right] \\
& \tau_{c}(t)=e^{-\lambda_{c} t}\left[T_{0}+\frac{\lambda_{c}}{l} \int_{0}^{t}\left(e^{\lambda_{c} s} \int_{0}^{l} \tau\left(r_{c}, z, s\right) \mathrm{d} z\right) \mathrm{d} s\right]
\end{aligned}
$$

and for a given $(\tau, \omega, \chi) \in \mathcal{B}$ we state the problem

$$
\begin{aligned}
\hat{\tau}_{t} & =A_{0}(\tau, \omega)\left[\Delta_{\chi} \hat{\tau}+\hat{\tau}_{y}\left(h_{0}-\chi\right) \psi_{\zeta \zeta}\right]+A_{1}(\tau, \omega) W(\tau, \omega) \\
-k \nabla_{\chi} \hat{\tau} \cdot \vec{n} & =\tilde{\Phi}(r, y, t)\left(\hat{\tau}-\tilde{T}_{e x t}\left(r, y, t ; \tau_{c}, \tau_{p}\right)\right) \quad \text { on } \Gamma \\
\hat{\tau}(r, t, 0) & =T_{0}
\end{aligned}
$$

where $\Delta_{\chi}$ and $\nabla_{\chi}$ are defined analagous to $\Delta_{h}$ with $h$ substituted by $\chi$. Existence and uniqueness of a solution in $\mathcal{H}^{2+\alpha, \frac{2+\alpha}{2}}$ of problem (5.5)-(5.6) is now assured by Theorem 5.3, p. 320 in [22].

From the uniform boundedness of $\tau$ the maximum principle easily gives $\frac{T_{e}}{2} \leqslant \hat{\tau} \leqslant 2 T_{0}$ for a sufficiently small $\theta$ depending only on $M_{1}, N_{1}, N_{2}$ and $Q$.

$\dagger$ The authors thank V.A. Solonnikov for this improvement of their result. 
The $\|\hat{\tau}\|_{\mathcal{H}^{\alpha, \frac{\alpha}{2}}}$ norm is estimated uniformly in terms of $M_{1}, N_{1}, N_{2}$ and $Q$ as stated in Theorem 9.1 , p. 341 in [22], so we can choose $M_{2}$ such that

$$
\|\hat{\tau}\|_{\mathcal{H}^{\alpha, \frac{\alpha}{2}}}>M_{2} \text {. }
$$

Finally the coefficients of (5.6) are estimated in the $\mathcal{H}^{1+\alpha, \frac{1}{2}+\frac{\alpha}{2}}$-norm by $M_{1}, N_{1}, N_{2}$ and $Q$ and Theorem 5.1 p. 320 in [22], again, gives an uniform estimate of $\|\hat{\tau}\|_{\mathcal{H}^{2+\alpha, 1+\frac{\alpha}{2}}}$ in the fixed interval $(0, \theta)$, so we can fix $M_{3}$ such that

$$
\|\hat{\tau}\|_{\mathcal{H}^{2+\alpha, 1+\frac{\alpha}{2}}}<M_{3}
$$

Now we define the second element of the triple $(\hat{\tau}, \hat{\omega}, \hat{\chi})$ :

$$
\hat{\omega}=\rho(\tau, \omega)\left\{(1-a) \int_{0}^{t} \rho^{a-1}(\tau, \omega) B(\tau)\left(w_{e q}-\omega\right)^{b} \mathrm{~d} t\right\}^{\frac{1}{1-a}}
$$

and we compute

$$
\hat{\omega}_{t}=\left(\rho_{T} \tau_{t}+\rho_{w} \omega_{t}\right) \frac{\hat{\omega}}{\rho}+\hat{\omega}^{\frac{a}{1-a}} \rho^{a-1} B\left(w_{e q}-\omega\right)^{b},
$$

from which we obtain

$$
\left|\hat{\omega}_{t}\right| \leqslant C_{1}\left(M_{3}+N_{2}\right) \theta^{\frac{1}{1-a}}+C_{2} \theta^{\frac{a}{1-a}}
$$

where $C_{i}$ denote uniform constants.

Likewise we obtain

$$
\left|\nabla_{h} \hat{\omega}\right| \leqslant C_{4}\left(M_{1}+N_{1}\right) \theta^{\frac{a}{1-a}} .
$$

Putting together the above estimates we realize that we can select the constants $N_{i}$ and $\theta$ so that $\hat{\omega}$ satisfies the same inequalities as $\omega$, including the constraint $\hat{\omega} \leqslant w_{\text {eq }}(\hat{\tau})-\varepsilon$. Next we complete the mapping $(\tau, \omega, \chi) \rightarrow(\hat{\tau}, \hat{\omega}, \hat{\chi})$ by means of

$$
2 \pi \int_{0}^{h_{0}} \int_{0}^{R} r \rho(\hat{\tau}, \hat{\omega})\left(1+\left(h_{0}-\hat{\chi}\right) \psi_{\zeta}\right) \mathrm{d} r \mathrm{~d} y=m,
$$

from which we realize that $\|\hat{\chi}\|_{\mathcal{H}} \frac{1}{2}+\frac{\alpha}{2}$ is estimated in terms of the Hölder coefficients of $\hat{\tau}(\cdot, t)$, $\hat{\omega}(\cdot, t)$ which as we have seen can be made as small as desired by reducing $\theta$. Therefore we obtain the last estimate

$$
\|\hat{\chi}\|_{\mathcal{H}^{\frac{1}{2}+\frac{\alpha}{2}}} \leqslant Q
$$

with arbitrary $Q$.

The last step of the proof consists of showing that the mapping $(\tau, \omega, \chi) \rightarrow(\hat{\tau}, \hat{\omega}, \hat{\chi})$ is contractive with respect to the sup-norm of the three elements. Denoting such a norm by $\|(\tau, \omega, \chi)\|_{0}$ we have to show that for any pair $\left(\tau_{1}, \omega_{1}, \chi_{1}\right),\left(\tau_{2}, \omega_{2}, \chi_{2}\right) \in \mathcal{B}$, we have

$$
\left\|\left(\hat{\tau}_{1}-\hat{\tau}_{2}, \hat{\omega}_{1}-\hat{\omega}_{2}, \hat{\chi}_{1}-\hat{\chi}_{2}\right)\right\|_{0} \leqslant \lambda\left\|\left(\tau_{1}-\tau_{2}, \omega_{1}-\omega_{2}, \chi_{1}-\chi_{2}\right)\right\|_{0}
$$


for some positive $\lambda<1$.

Writing (5.14) for $\left(\hat{\tau}_{1}, \hat{\omega}_{1}\right)$ and $\left(\hat{\tau}_{2}, \hat{\omega}_{2}\right)$ and subtracting we obtain, by using the regularity of $\rho$ and $F$, the estimate

$$
\sup _{D_{\theta}}\left|\hat{\chi}_{1}-\hat{\chi}_{2}\right| \leqslant C_{\chi} \sup _{D_{\theta}}\left\{\left|\hat{\tau}_{1}-\hat{\tau}_{2}\right|+\left|\hat{\omega}_{1}-\hat{\omega}_{2}\right|\right\}
$$

where by means of the boundedness of $\rho, B, w_{e q}$ in (5.10), we obtain

$$
\sup _{D_{\theta}}\left|\hat{\omega}_{1}-\hat{\omega}_{2}\right| \leqslant \theta C_{\omega} \sup _{D_{\theta}}\left\{\left|\tau_{1}-\tau_{2}\right|+\left|\omega_{1}-\omega_{2}\right|\right\} .
$$

Finally, by applying Theorem 2.2, p. 15 of [22] to the parabolic equation we can obtain, for $\hat{\tau}_{1}-\hat{\tau}_{2}$ (which has zero initial data), guarantees that we can estimate from the above the sup-norm of $\mid \hat{\tau}_{1}-$ $\hat{\tau}_{2} \mid$ with $\sup _{D_{\theta}}\left\{\left|\tau_{1}-\tau_{2}\right|+\left|\omega_{1}-\omega_{2}\right|\right\}$ times a decreasing function of time.

$$
\sup _{D_{\theta}}\left|\hat{\tau}_{1}-\hat{\tau}_{2}\right| \leqslant \mathcal{F}(\theta) \sup _{D_{\theta}}\left\{\left|\tau_{1}-\tau_{2}\right|+\left|\omega_{1}-\omega_{2}\right|\right\}
$$

with $\mathcal{F}(0)=0$.

Putting back (5.18) and (5.19) in (5.17) we obtain that (5.16) can be satisfied for $\theta$ sufficiently small. This concludes the proof thanks to the Banach-Caccioppoli contraction lemma being $\mathcal{B}$ closed in the space of continous functions.

\section{Continuation of the solution}

So far we have solved the problem using (2.4) concerning the threshold $w_{e q}(T)$. We have shown that a solution exists locally in time, as long as that threshold is not reached. Indeed, when $w$ crosses the value $w_{e q}(T)$ there is a discontinuity deriving from a crude representation of the switch of the crystallization mechanism. The physics of such a change is not completely clear and it may well be that it occurs only in a cooling regime $\left(T_{t}<0\right)$, due to secondary crystallization within the spherulites as we explained in section 2. As was been pointed out there, a smooth transition between (2.4) and the obstacle $w=w_{e q}$ might be acceptable. Next we present one way for such a change. It seems quite reasonable to slightly modify the model when approaching $w_{e q}$ in order to eliminate that artificial singularity. This can be achieved by redefining $w_{t}$ for values of $w$ in the interval $\left(w_{e q}-\varepsilon, w_{e q}\right)$. Setting

$$
F_{\varepsilon}(T)=-\left(w_{e q}-\varepsilon\right) \operatorname{div} \vec{V}+W\left(T, w_{e q}-\varepsilon\right)
$$

and

$$
z_{\varepsilon}(T, w)=\frac{w_{e q}-w}{\varepsilon}, \quad z_{\varepsilon}(T, w) \in[0,1]
$$

for all $w$ in the interval above, we write the following evolution equation.

$$
\varepsilon z_{\varepsilon t}+z_{\varepsilon}^{b}\left[F_{\varepsilon}(T)-\frac{\partial}{\partial t} w_{e q}(T)\right]=0, \quad z_{\varepsilon}\left(t^{*}\right)=1
$$


$\left(t^{*}\right.$ being such that $\left.w\left(\cdot, t^{*}\right)=w_{e q}\left(T\left(\cdot, t^{*}\right)\right)-\varepsilon\right)$ which gives,

$$
z_{\varepsilon}=\left\{1-\frac{1-b}{\varepsilon}\left[\int_{t^{*}}^{t} F_{\varepsilon}(T(\cdot, \tau)) \mathrm{d} \tau-\left(w_{e q}(T(\cdot, t))-w_{e q}\left(T\left(\cdot, t^{*}\right)\right)\right)\right]\right\}^{\frac{1}{1-b}}
$$

as long as $z>0$ (note that $z=0$ is reached in a finite time), and finally

$$
\begin{aligned}
w(\cdot, t)=w_{e q}(T(\cdot, t))-\varepsilon & \\
& \left\{1-\frac{1-b}{\varepsilon}\left[\int_{t^{*}}^{t} F_{\varepsilon}(T(\cdot, \tau)) \mathrm{d} \tau-\left(w_{e q}(T(\cdot, t))-w_{e q}\left(T\left(\cdot, t^{*}\right)\right)\right)\right]\right\}^{\frac{1}{1-b}} .
\end{aligned}
$$

The source term in the heat balance equation is proportional to

$$
\begin{aligned}
& w_{t}= \frac{\partial}{\partial t} w_{e q}(T)-\varepsilon \frac{\partial z}{\partial t}= \\
& w_{e q}^{\prime}(T) T_{t}+\left\{1-\frac{1-b}{\varepsilon}\left[\int_{t^{*}}^{t} F_{\varepsilon}(T(\cdot, \tau)) \mathrm{d} \tau-\left(w_{e q}(T(\cdot, t))-w_{e q}\left(T\left(\cdot, t^{*}\right)\right)\right)\right]\right\}^{\frac{1}{1-b}} \\
& \\
& {\left[F_{\varepsilon}(T(\cdot, t))-w_{e q}^{\prime}(T)\right] T_{t}=\mathcal{A}_{3} w_{e q}^{\prime}(T) T_{t}+\mathcal{A}_{4} . }
\end{aligned}
$$

Note that $\mathcal{A}_{3} \in[0,1]$, and $w_{e q}^{\prime}<0$, so that the equation replacing (5.1) remains parabolic.

In conclusion, the heat balance equation has the form (5.1) as long as $w<w_{e q}-\varepsilon$, otherwise the evolution of the temperature field is given by (2.1) with $w_{t}$ replaced by (6.6).

At this point is not difficult to obtain the continuation of the solution for all times by means of obvious modifications of the proof of Theorem 5.1. Thus we can state the following

THEOREM 6.1 The problem with a modified model for the switch from growth regime to the constrained regime $w=w_{e q}(T)$ has an unique classical solution in any time interval.

\section{Numerical results and physical implications}

As already mentioned in the introduction, the model presented here helped to point out some interesting features of the physical phenomenon as well as a good agreement with experimental data (see Fig. 3 for a comparison of the numerical- $(P, V, T)$ diagram with the experiment in the temperature range where the 'unconstrained' crystallization is active).

In fact, the numerical simulations have been performed with a more complex model than the one presented here (see [16]) where convective terms are not neglected and the Stokes equation has to be solved in each time step.

An important result obtained from such simulations was that all the simplifications adopted in the model are largely justified. At the same time the model proves to be able to describe the evolution of quantities which are relevant to the phenomenon, such as the thermal field and distribution of the crystal volume fraction, as well as the local contraction rate and the overall shrinkage of the sample (see Figs 4 and 5). In particular, we emphasize that even in cases where radial flux is present, its contribution to convection is negligibly small. 


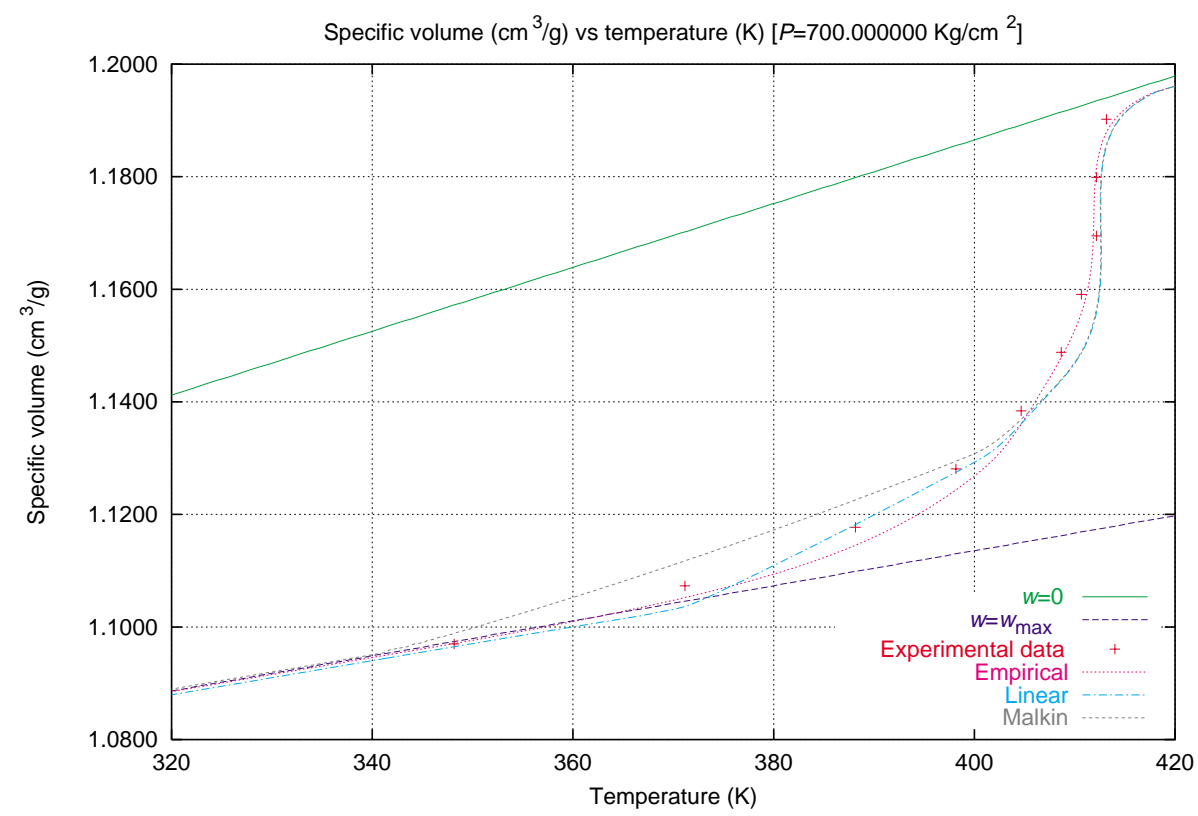

FIG. 3. Specific volume vs temperature diagram for a pressure of $700 \mathrm{Kg} / \mathrm{cm}^{2}$, different models for the evolution of $w_{e q}$ are presented.

\section{Acknowledgements}

This work was partially supported by Federchimica, ASI (Italian Space Agency) and CNR (Italian Research Council).

\section{REFERENCES}

1. Andreucci D., Fasano A., Gianni R., Primicerio M., \& Ricci R. Modelling nucleation in crystallization of polymers. In NiezGodKA, M. \& Strzelecki, P. (eds), Conference on Free Boundary Problems, Zakopane, 1995, pp. 359-367.

2. Andreucci, D., Fas ano, A., Paolini, M., Primicerio, M., \& Verdi, C. Numerical simulation of polymer crystallization. Math. Models Methods Appl. Sci. 4, (1994) 135-145.

3. Andreucci, D., Fasano, A., \& Primicerio, M. A mathematical model for non isothermal crystallization, Proceedings of Research Institute for Mathematical Sciences, no. 755, 1991, Kyoto, pp. $112-120$.

4. Andreucci, D., Fasano, A., \& Primicerio, M. On a mathematical model for the crystallization of polymers. In WACKER, H. \& ZULEHNER, W. (eds), Fourth European Conference on Mathematics in Industry, Teubner Stuttgart, 1991, pp. 3-16.

5. Andreucci, D., Fasano, A., Primicerio, M., \& Ricci, R. Mathematical models in polymer crystallization. Surv. Math. Ind. 6, (1996) 7-20.

6. Astarita, G. \& Ocone, R. Continuous and discontinuous models for transport phenomena in polymers. AIChE. J. 33, (1987) 423-435. 
7. Beghishev, V. P., Bolgov, S. A., Keapin, I. A., \& Malkin, A. Y. General treatment of polymer crystallizantion kinetics. Part 1: a new macrokinetic equation and its experimental verification. Polym. Eng. Sci. 24, (1984) 1396-1401.

8. Berger, J. \& Schneider, W. A zone model of rate controlled solidification. Plast. Rubber Process. Appl. 4, (1986) 127-133.

9. Capasso, V., Micheletti, A., De Giosa, M., \& Mininni, R. Stochastic modelling and statistics of polymer crystallization processes. Surv. Math. Ind. 6, (1996) 109-132.

10. Caselli, R., Mazzullo, S., Paolini, M., \& Verdi, C. Models, experiments and numerical simulation of isothermal crystallization of polymers. In FASANO, A. \& PRIMICERIO, M. (eds) European Consortium for Mathematics in Industry VII, Teubner Stuttgart, 1993, pp. 167-174.

11. ChOw, T. S. Molecular kinetic theory of the glass transition. Polym. Eng. Sci. 24, (1984) 1079-1086.

12. Clark, E. J. \& Hoffman, J. D. Regime III crystallisation in polypropylene. Macromolecules 17 , (1984) 878-885.

13. De Luigi, C., Corrieri, R., \& Mazzullo, S. Modello matematico di cristallizzazione isobara, non isoterma, di polipropilene, XII convegno italiano di scienza e tecnologia delle macromolecole, 1995, Altavilla Milicia.

14. Eder, G., Janeschitz-Kriegl, H., \& Liedauer, S. Crystallization processes in quiescent and moving polymer melts under heat transfer conditions. Prog. Polym. Sci. 15, (1990) 629-714.

15. FASANO, A. Modelling the solidification of polymers. An example of an ecmi cooperation, ICIAM 91 (R.E.O’Malley, ed.), 1991, Washington.

16. Fasano, A., Mancini, A., \& Mazzullo, S. Non isothermal crystallization of polypropilene. In Fas Ano,A. (ed.), Complex Flows and Industrial Processes, Birköser, (1999), to appear.

17. FASANO, A. \& PRIMICERIO, M. On mathematical models for nucleations and crystal growth processes. In LIONS, J. L. \& BAIOCCHI C. (eds), Boundary Value Problems for PDE's and Applications, pp. 351358. Masson-Paris (1993).

18. FASANO, A. \& PRIMICERIO, M. On a class of travelling wave solutions to phase change problems with an order parameter, Workshop on non linear analysis an applications, 1995, Warsaw, pp. 113-123.

19. Fasano, A. \& Primicerio, M. An analysis of phase transition models. EJAM 7, (1996) 1-12.

20. Kamal, M. R. \& Lafleur, P. G. A structure oriented simulation of the injection molding of viscoelastic crystalline polymers. Part 1: model with fountain flow, packing, solidification. Polym. Eng. Sci. 26, (1986) 92-102.

21. Kovars KIJ, A. L. High-pressure Chemistry and Physics of Polymers (Compressibility of Polymers). CRC Press, London (1994).

22. Ladyzenskaja, O. A., Solonnikov, V. A., \& Ural'Ceva, N. N. Linear and quasi-linear equations of parabolic type, Translations of Mathematical Monographs. 23. Providence, RI, American Mathemtical Society (1968).

23. MANCINI, A. Non isothermal crystallization of polypropilene: Numerical approach, to appear.

24. Mancini, A. Processo di cristallizzazione non isoterma di polipropilene in condizioni isobare. Internal report, Dip. Matematica "U.Dini” - Università degli Studi di Firenze (1997).

25. MANCINI, A. A model for the crystallization of polypropylene under pressure. In LEIF ARKERYS JÖRAn Bergh, P. B. \& Pettersson R. (eds), European Consortium for Mathematics in Industry 98, Göteborg, 1999, pp. 146-153.

26. Mazzullo, S., Corrieri, R., \& De Luigi, C. Mathematical model for isobaric non-isothermal crystallization of polypropylene. In BRONS, M. (ed.), European Consortium for Mathematics in Industry 96, no. 307, B.G. Teubner-Stuttgart, 1997.

27. Mazzullo, S., Paolini, M., \& Verdi, C. Polymer crystallization and processing: free boundary problems and their numerical approximation. Math. Eng. Ind. 2, (1989) 219-232.

28. Micheletti, A. Problemi di geometria stocastica nei processi di cristallizzazione di polimeri. aspetti 
modellistici, statistici e computazionali. PhD thesis, Università degli Studi di Milano (1997).

29. SolonNikov, V. A. Solvability of the classical initial-boundary-value problems for the heat conduction equation in a dihedral angle. J. Sov. Math. 32, (1986) 526-546, (translated from Russian).

30. Tobin, M. C. Theory of phase transition kinetics with growth site impingement. J. Polym. Sci. Polym. Phys. Ed. 12, (1974) 394-406. 


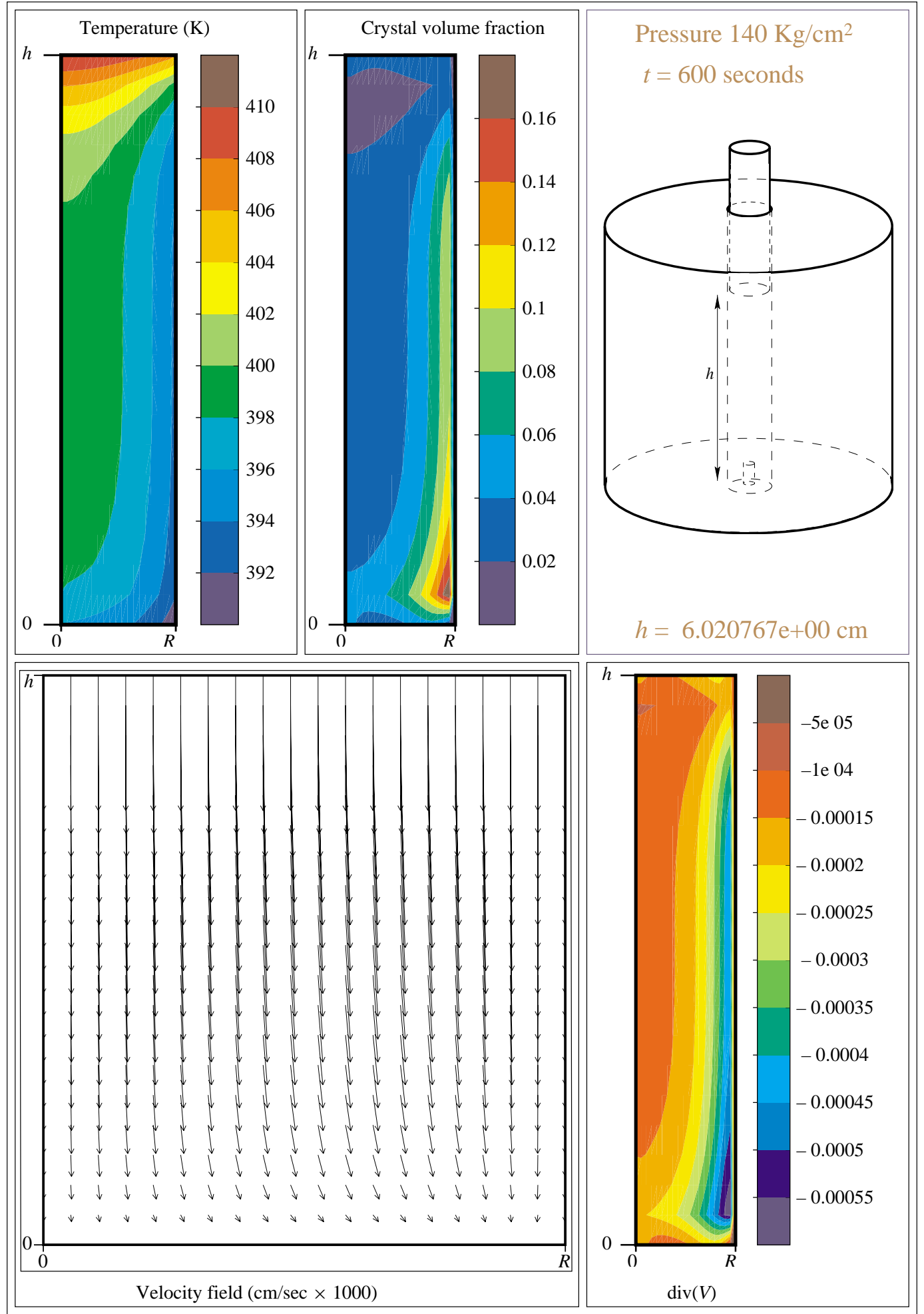

FIG. 4. $600 \mathrm{sec}$ 


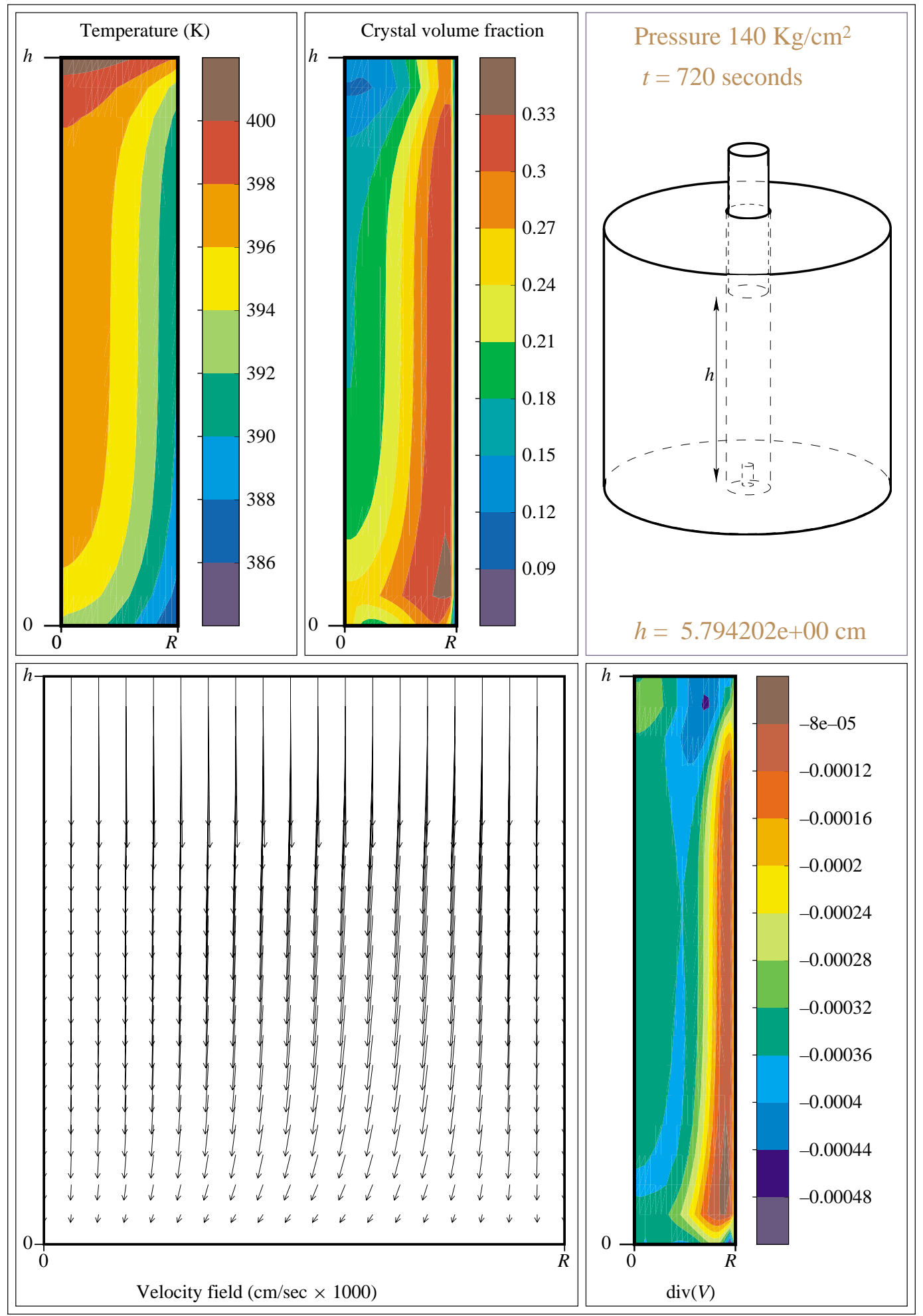

FIG. 5. $720 \mathrm{sec}$ 